Sergej Springer, Niclas Heidenreich, Norbert Stock, Leo van Wüllen, Klaus Huber, Stefano Leoni and Michael Wiebcke*

\title{
The ZIF system zinc(II) 4,5-dichoroimidazolate: theoretical and experimental investigations of the polymorphism and crystallization mechanisms
}

DOI 10.1515/zkri-2016-1968

Received June 2, 2016; accepted August 15, 2016; published online September 17, 2016

Abstract: In this report, we summarize our theoretical and experimental investigations on the zeolitic imidazolate framework (ZIF) system [Zn(dcim) $\left.{ }_{2}\right](\mathrm{dcim}=4,5$-dichloroimidazolate) that have been published recently. These comprise: (1) a theoretical study on hypothetical conformational $\left[\mathrm{Zn}(\mathrm{dcm})_{2}\right]$-SOD polymorphs with the same underlying sodalite (SOD) topology but distinct dcim linker orientations, (2) a synthetic work that resulted in the experimental realization of the most stable predicted (trigonal) SOD-type framework conformer and improved synthetic protocols for a previously discovered cubic SOD-type material, (3) a detailed structural analysis of the trigonal and cubic SOD-type materials, (4) a comparative characterization of the SOD-type materials by gas physisorption measurements, (5) a synthetic work that resulted in the discovery of a complete series of intermediate frameworks with the trigonal and cubic SOD-type materials as the end members, and (6) time-resolved insitu light and stopped-flow synchrotron small-angle and wide-angle X-ray scattering experiments on the rapid crystallization of the RHO-type polymorph (ZIF-71). In addition, we report as yet unpublished work, concerning time-resolved in-situ angular-dispersive synchrotron X-ray diffraction experiments on RHO-/SOD-type phase

*Corresponding author: Michael Wiebcke, Institut für Anorganische Chemie, Leibniz Universität Hannover, Callinstrasse 9, 30167 Hannover, Germany, E-mail: michael.wiebcke@acb.uni-hannover.de Sergej Springer: Institut für Anorganische Chemie, Leibniz Universität Hannover, Callinstrasse 9, 30167 Hannover, Germany Niclas Heidenreich and Norbert Stock: Institut für Anorganische Chemie, Christian-Albrechts-Universität zu Kiel, Max-Eyth-Strasse 2, 24118 Kiel, Germany

Leo van Wüllen: Institut für Physik, Universität Augsburg, Universitätsstrasse 1, 86159 Augsburg, Germany

Klaus Huber: Department Chemie, Universität Paderborn, Warburger Strasse 100, 33098 Paderborn, Germany

Stefano Leoni: School of Chemistry, Cardiff University, Main Building, Park Place, Cardiff CF10 3AT, UK selection via the coordination modulation approach during competitive formation of the RHO-type and SOD-type materials.

Keywords: crystallization mechanism; density functional theory; in-situ investigations; polymorphism; zeolitic imidazolate framework.

\section{Introduction}

Metal imidazolates of divalent metal cations (e.g. Zn, Co) form dense (non-porous) and open (porous) framework structures with underlying 3-dimensional tetrahedral networks (zeolitic imidazolate frameworks, ZIFs) with properties [1-3] that make them potentially useful for applications in many different fields ranging from separation [4] and catalysis [5] to drug delivery [6]. However, discovery of theoretically predicted and potentially existing new ZIFs still proceeds mainly on a "trial-and-error" basis [7], because synthesis-structure relationships and mechanisms of crystallization are poorly understood $[1,8,9]$.

Since the publication of our first report in late 2014 [1], a number of remarkable papers have appeared on the prediction of hypothetical and potentially accessible structures and the mechanisms of crystallization. Going beyond earlier theoretical work based on density functional theory (DFT) to calculate relative framework stabilities at $0 \mathrm{~K}[10,11]$, two research groups used force field-based molecular simulation methods to investigate for the first time the influence of solvent, temperature and pressure on the stability of ZIF frameworks with different topologies. According to Gee and Sholl [12], a solvent (methanol) can stabilize a porous [ $\operatorname{Zn}(2-$ $\mathrm{mim})_{2}$ ] polymorph (2-mim $=2$-methylimidazolate) by $c a$. $5-10 \mathrm{~kJ} \cdot \mathrm{mol}^{-1}$ (at $298 \mathrm{~K}$ ) and change the energetic order of the frameworks compared to the order of the solventfree forms. Bouessel du Bourg et al. [13] have estimated that only ten out of a total of 18 considered, experimentally known and hypothetical $\left[\mathrm{Zn}(\mathrm{im})_{2}\right]$ polymorphs 
(im =imidazolate) are stable at relevant temperatures (>300 K). They have also demonstrated the stabilizing effect of a non-polar "solvent" (methane) on an unstable framework due to space filling and have predicted that some experimental as-made (solvent-containing) ZIFs are not stable upon guest removal.

A detailed in-situ electro spray ionization mass spectrometry (ESI-MS) investigation on the formation of [Zn(2-mim) $\left.{ }_{2}\right]$-SOD (ZIF-8) particles in comparatively dilute methanolic solutions has been reported by Lim et al. [14], providing for the first time information about molecular assembly during pre-nucleation and nucleation stages. They demonstrated experimentally the formation and depletion of multinuclear complexes (oligomers) with up to seven zinc ions by complex formation, complex deprotonation and ligand exchange reactions, in agreement with an earlier proposal [15], and detected tetramers that could form four-membered rings, a basic unit of the SODtype ZIF-8 framework. The growth of ZIF-8 particles was successfully monitored by Patterson et al. [16] in a careful in-situ liquid cell transmission electron microscopy (LCTEM) study. It was demonstrated that particle growth is not a coalescence process, confirming the results of earlier in-situ small-angle and wide-angle X-ray scattering (SAXS/WAXS) experiments [17], and that particle growth is surface-reaction limited and not diffusion limited. The topotactic transformation of a layered zinc 2-methylimidazolate (ZIF-L) into ZIF-8 was investigated using various ex-situ methods as well as in-situ liquid-state ${ }^{1} \mathrm{H}$ NMR spectroscopy by Low et al. [18]. Analysis of the insitu NMR data suggested that the solid-state reaction followed a geometrical contraction model (R2), in line with microscopic observations. Furthermore, Self et al. [19] have discovered for a RHO-type hetero-ZIF made from two different linkers (2-nitroimidazolate and purinate) an interesting reversed crystal growth mechanism. It starts from a disordered aggregate of precursor materials that undergoes a surface recrystallization process with subsequent growth from the crystalline rhombic dodecahedral shell to the disordered core.

Herein, we summarize our recent theoretical and experimental work on the $\left[\mathrm{Zn}(\mathrm{dcim})_{2}\right]$ system (dcim $=4,5$-dichloroimidazolate), which we have chosen as a model ZIF system for investigations of the polymorphism and crystallization mechanisms. Further details can be found in two recent publications [20,21]. Some previous studies on the $\left[\mathrm{Zn}(\mathrm{dcim})_{2}\right]$ system [22] had already been summarized elsewhere [1]. In addition, we present as yet unpublished work. This concerns in-situ synchrotron X-ray diffraction (XRD) experiments on phase selection via the coordination modulation approach.

\section{Energy landscape of the $\left[\mathrm{Zn}(\mathrm{dcim})_{2}\right]$ system considering topological and conformational polymorphs}

Our previous theoretical investigations on the $\left[\mathrm{Zn}(\mathrm{dcim})_{2}\right]$ system had considered synthetically realized and hypothetical framework structures based on different underlying 3-dimentional tetrahedral networks (topological polymorphs) [22]. That theoretical work has been extended to additionally include hypothetical frameworks with SOD topology exhibiting different dcim linker orientations (conformational [Zn(dcim) 2 -SOD polymorphs). Difficulties encountered during structural analysis of a previously discovered cubic [Zn(dcim) ${ }_{2}$-SOD material [22] forced us to search for further structural models (see below). Figure 1 shows an energy-density diagram for topological and conformational $\left[\mathrm{Zn}(\mathrm{dcm})_{2}\right]$ polymorphs obtained by periodic DFT calculations that took van der Waals interactions into account (rVV10 non-local density functional [23]).

The frameworks of the four conformational SODtype polymorphs, SOD-I to SOD-IV, that have been generated and optimized by DFT calculations are illustrated in Figures 2 and 3.

It can be seen that the four SOD-type frameworks differ considerably in their conformations as expressed by the orientations (twists) of the dcim linkers as well as the rotations (tilts) of the $\mathrm{ZnN}_{4}$ tetrahedra. They are also energetically destabilized by $<40 \mathrm{~kJ} \cdot \mathrm{mol}^{-1}$ compared to the dense (non-porous) lcs-type polymorph. The latter, experimentally known polymorph (ZIF-72) [25] represents the ground state among the considered structures. Framework SOD-I

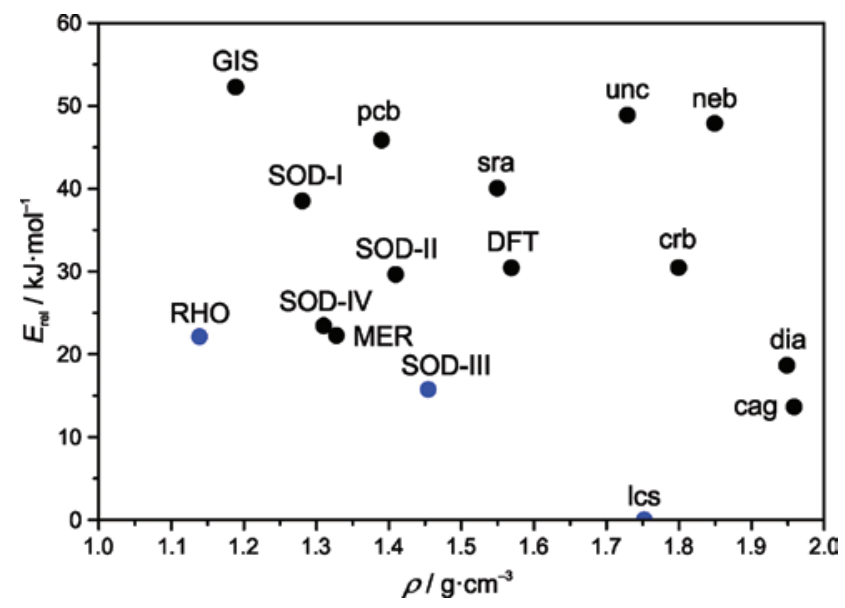

Fig. 1: Energy-density diagram for topological and conformational [Zn(dcim) $)_{2}$ polymorphs. Black and blue dots indicate hypothetical and experimentally realized polymorphs, respectively. Reproduced from Ref. [20] with permission from the Royal Society of Chemistry. 
SOD-I
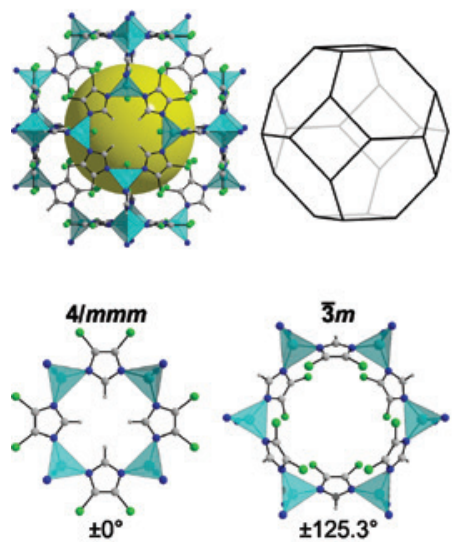

SOD-II
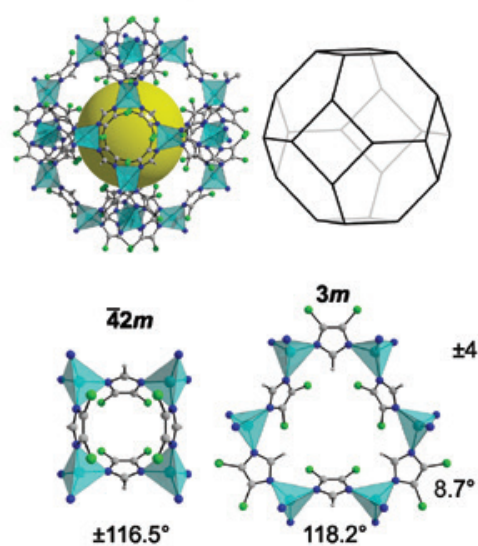

SOD-III
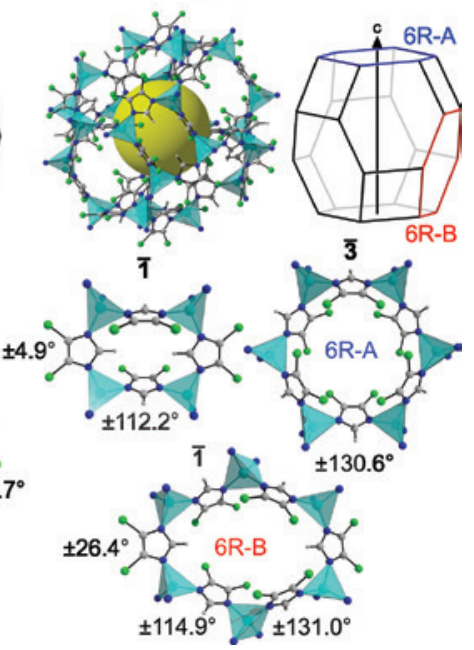

Fig. 2: Structural diagrams of the SOD-I (cubic $I m \overline{3} m$ ), SOD-II (cubic $1 \overline{4} 3 m$ ) and SOD-III (trigonal $R \overline{3}$ ) conformational polymorphs that have been optimized by DFT. The unique $\left[4^{6} \cdot 6^{8}\right]$ truncated octahedral cages and unique four- and six-membered rings are shown. The point group symmetries and twist angles of the dcim linkers with respect to the planes of the four- and six-membered rings are indicated. Color code: cyan, $\mathrm{ZnN}_{4}$ tetrahedra; blue, $\mathrm{N}$; gray, $\mathrm{C}$ and $\mathrm{H}$; green, $\mathrm{Cl}$. Reproduced from Ref. [20] with permission from the Royal Society of Chemistry.
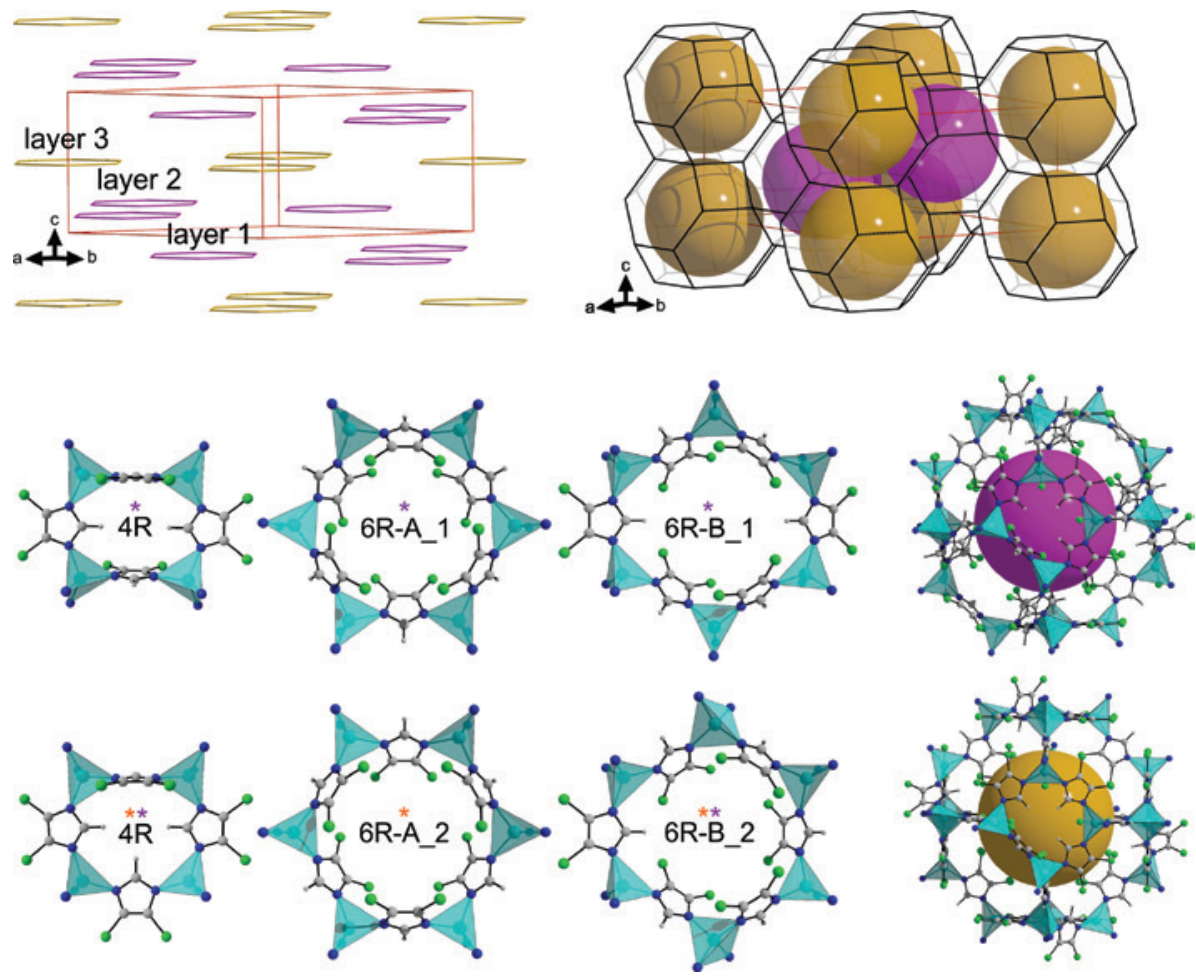

Fig. 3: Structural diagram of the SOD-IV conformational polymorph (trigonal $P \overline{3} m 1$ ) that has been optimized by DFT. Upper panel: ABC stacking of 2-dimensional periodic building units (non-connected planar six-rings) of the SOD topology [24] with indication of the conformations of the unique 6R-A rings (pink, conformation as in SOD-III; orange, conformation as in SOD-I) and SOD topology with indication of the two unique $\left[4^{6} \cdot 6^{8}\right]$ cages (pink and orange spheres). Lower panel: unique cages and four- and six-membered rings. The colored asterisks in the four- and six-membered rings indicate by which cages the rings are shared. Color code: cyan, $\mathrm{ZnN}_{4}$ tetrahedra; blue, $\mathrm{N}$; gray, $\mathrm{C}$ and $\mathrm{H}$; green, $\mathrm{Cl}$. 
in cubic space group $\operatorname{Im} \overline{3} m$ represents the fully expanded conformational state with the lowest density [26], while the denser conformers SOD-II (cubic I $\overline{4} 3 \mathrm{~m}$ ) and SOD-III (trigonal $R \overline{3}$ ) show different kinds of distortion from the maximal-symmetry framework. The latter two conformers resemble the framework conformations found in the low- and high-pressure forms of ZIF-8 [27-31] and guestfree/guest-containing forms of $\left[\mathrm{Zn}(\mathrm{bim})_{2}\right]$-SOD (ZIF-7) with the benzimidazolate (bim) linker [28, 32-34], respectively. It is interesting to note that the analogs of all three [Zn(dcim) $\left.)_{2}\right]$-SOD frameworks are known in silica sodalites $[35,36]$. Conformer SOD-IV (trigonal $P \overline{3} m 1$ ) represents one example out of an infinite number of hypothetical periodic as well as non-periodic or defective intermediate frameworks of SOD-I and SOD-III that can be readily theoretically generated via formal concerted orientational movements of certain dcim linkers as described in detail elsewhere [20].

The large differences in the framework conformations of the SOD-type polymorphs considered here suggest that possible interconversions between any pair of frameworks are unlikely to take place via displacive transition pathways, because certain linkers would necessarily have to move concertedly through the comparatively small apertures of the six-membered rings and would thereby encounter strong steric hindrance between the chlorine substituents at the imidazolate rings. Rather possible interconversions should be reconstructive in nature, and it is therefore reasonable to expect that the framework conformers represent local minima in the energy landscape, which are separated from each other by high energy barriers, in principal comparable to the situation for topological polymorphs. Thus, our theoretical studies predict that the $\left[\mathrm{Zn}(\mathrm{dcim})_{2}\right]-S O D$ framework potentially exhibits a great static conformational variety with the possibility of synthetically accessing some of the predicted conformational polymorphs as co-existing phases at a given set of parameters (e.g. ambient temperature and pressure), in particular those frameworks that are not significantly higher in energy than the experimentally known RHOtype polymorph (ZIF-71) [25]. It is important to distinguish this static conformational variety from the comparatively small dynamic conformational changes occurring in response to external stimuli (temperature, pressure, guest adsorption/desorption) in heavily investigated [37] flexible ZIF frameworks (e.g. ZIF-7 [33, 34] and ZIF-8 [29-31]), which cannot give rise to the co-existence of phases.

Conformational polymorphism in ZIFs had been considered before only by Baburin and Leoni [38] in a theoretical study of the $\left[\mathrm{Zn}(\mathrm{im})_{2}\right]$-dia framework with diamond topology.

\section{Experimental realization of a predicted trigonal [Zn(dcim) $\left.)_{2}\right]-S O D$ polymorph}

Realization of the trigonal SOD-III polymorph, the most stable amongst the SOD-type conformers, was only possible after laborious exploratory synthetic work. First hints at this conformer were obtained when inspecting powder XRD patterns of products recovered from reactions performed in aqueous ammonia. This represented a modification to previous synthetic work [39]. Inspection indicated some splitting of the most intense reflection in the powder XRD pattern of a previously discovered cubic [Zn(dcim) $)_{2}$-SOD material [22]. Those syntheses could be subsequently modified by replacing the solvent (DMF for 1-propanol) and modulator (1-methylimidazole for $\mathrm{NH}_{3}$ ) to successfully recover the SOD-III polymorph as a pure phase, as verified by full Rietveld refinement against powder XRD data from as-made (DMF-containing) as well as activated (solvent-free) samples. Removal of DMF was found to occur with retention of the framework symmetry $(R \overline{3})$ and a small decrease in cell volume. In contrast, for isostructural ZIF-7 it was reported that removal of DMF is accompanied by a displacive framework transition from ZIF-7-I (trigonal $R \overline{3}$ ) to ZIF-7-II (triclinic $P \overline{1}$ ) and a small increase in cell volume [33], revealing subtle differences in the (dcim vs. bim) inter-linker interactions.

\section{Improved synthesis and structural analysis of a previously discovered cubic $\left[\mathrm{Zn}\left(\right.\right.$ dcim $\left._{2}\right]$-SOD material}

Replacement of the solvent (THF for 1-propanol) and the zinc salt (acetate for nitrate) in previous 1-methylimidazole-modulated syntheses was found to yield the same cubic $\left[\mathrm{Zn}(\mathrm{dcim})_{2}\right]$-SOD material with improved crystallinity, which diffracted X-rays to higher $2 \theta$ angles. Moving on from an initial not completely satisfactory analysis [22], such samples were used for attempting structural solution and refinement on the basis of powder XRD data supported by solid-state NMR spectroscopy. The ${ }^{1} \mathrm{H}$ MAS NMR and ${ }^{13} \mathrm{C}\left\{{ }^{1} \mathrm{H}\right\} \mathrm{CP} / \mathrm{MAS}$ NMR spectra of the cubic [Zn(dcim) $\left.)_{2}\right]$ SOD material resemble the corresponding spectra of the trigonal SOD-III polymorph (Figure 4). Furthermore, it was noticed that the cell metric of the SOD-III polymorph is pseudo-body-centered cubic and closely resembles that of the (body-centered) cubic $\left[\mathrm{Zn}(\mathrm{dcim})_{2}\right]-\mathrm{SOD}$ 

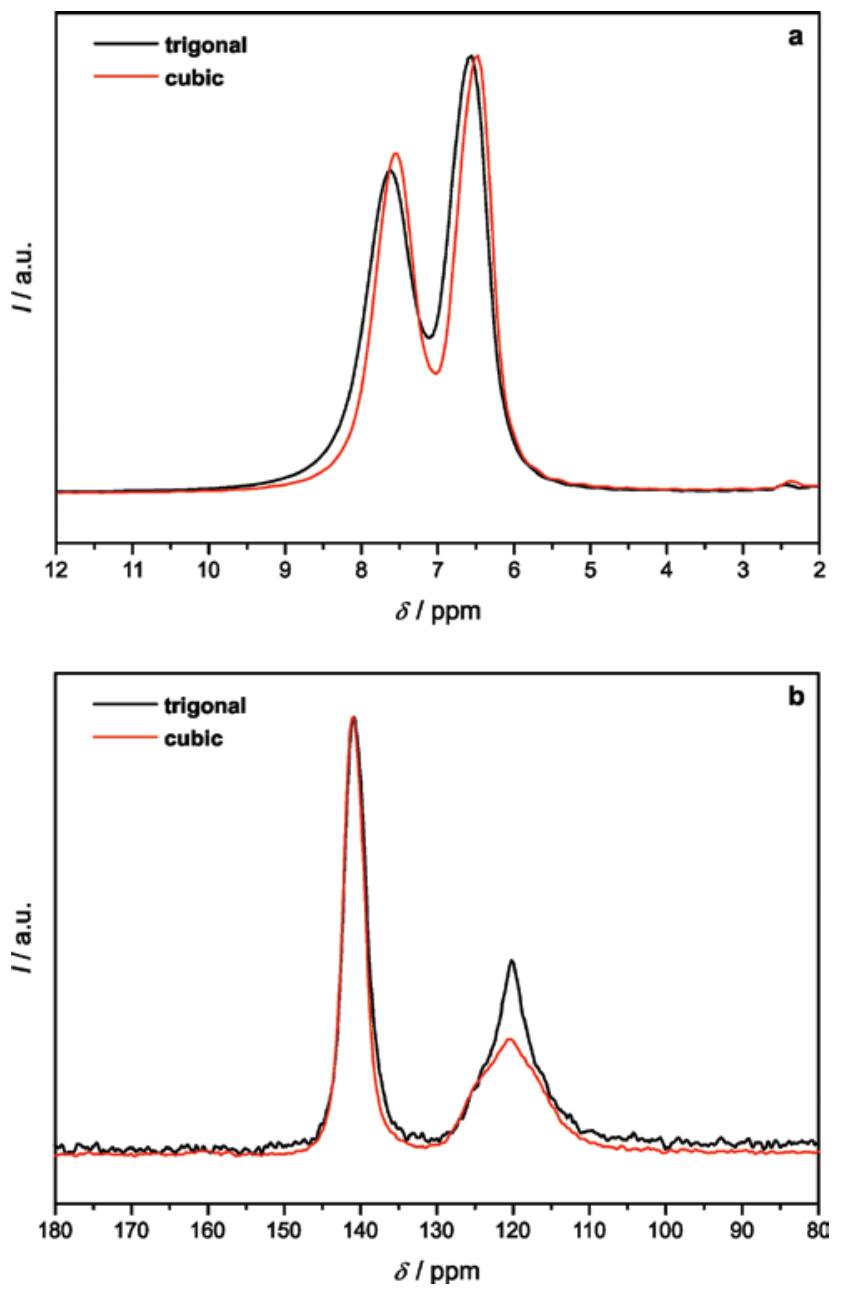

Fig. 4: (a) ${ }^{1} \mathrm{H}$ MAS NMR and (b) ${ }^{13} \mathrm{C}\left\{{ }^{1} \mathrm{H}\right\} \mathrm{CP} / \mathrm{MAS}$ NMR spectra of the trigonal (black) and cubic (red) SOD-type materials. Reproduced from Ref. [20] with permission from the Royal Society of Chemistry.

material. Due to these observations, a Rietveld refinement against powder XRD data from an activated sample of the cubic [ $\left.\mathrm{Zn}(\mathrm{dcim})_{2}\right]-S O D$ material was performed in $R \overline{3}$, starting with a SOD-III structural model deformed to a cubic metric, which yielded a quite nice Rietveld plot (Figure 5a).

However, under the same ambient conditions the solvent-free [Zn(dcim) $)_{2}$-SOD framework cannot exist with two different (rhombohedral vs. cubic) metrics and, indeed, closer inspection of the Rietveld plot reveals that a weak reflection (indicated by an arrow in Figure 5a) is not well modeled (see also the differences in line shapes in the ${ }^{13} \mathrm{C}$ NMR spectra in Figure 4, suggesting that some structural differences must exist).

A satisfactory Rietveld plot (Figure 5b) was finally obtained in cubic $\operatorname{Im} \overline{3} m$ with a structural model that contains two unique sites for the linkers in the crystallographic asymmetric unit, having almost even (refined)

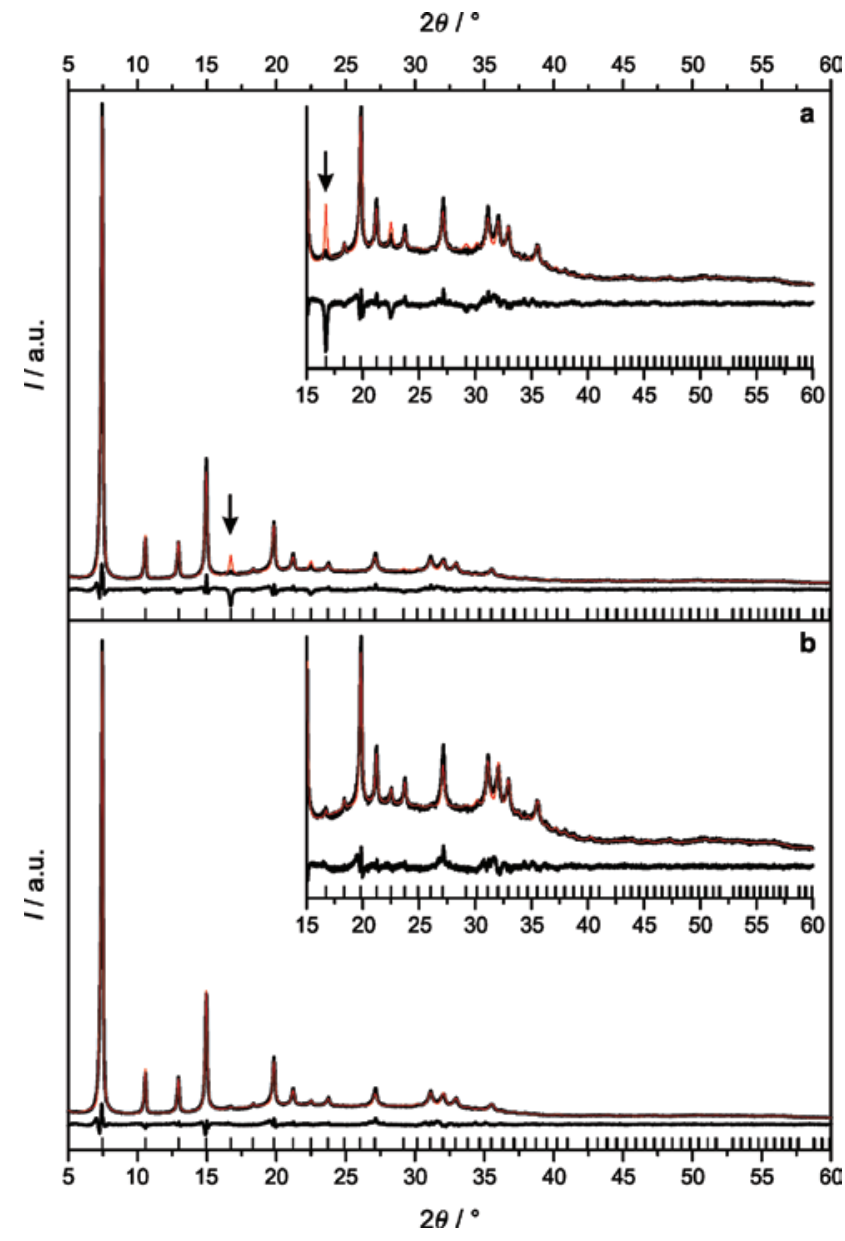

Fig. 5: (a) Rietveld refinement plot for the solvent-free cubic SODtype material in space group $R \overline{3}$ with the ordered SOD-III framework model. The arrow indicates the poorly fitted 310 reflection. (b) Final Rietveld refinement plot for the solvent-free cubic SOD-type material in $I m \overline{3} m$ with orientational disorder of the dcim linkers. Experimental and calculated profiles are shown in black and red, respectively; also shown are the difference plots and markers for allowed Bragg peaks. Reproduced from Ref. [20] with permission from the Royal Society of Chemistry.

statistical occupancy factors. This is in line with the equal intensities of the two signals in the ${ }^{1} \mathrm{H}$ NMR spectrum. The statistics of the in-plane and out-of-plane linker orientations in the four- and six-membered rings (Figure 6) is close to that in the trigonal SOD-III conformer, but differs from that in the other considered SOD-type polymorphs.

This suggests that orientational linker disorder is not random. Rather, we hypothesize that the crystals consist of nanoscopic domains of the SOD-III conformer with $R \overline{3}$ symmetry that are deformed to cubic metric and aligned with their $\overline{3}$ axes along the cubic $<111>$ directions. The energetic cost for deforming the trigonal SOD-III structure to a cubic metric is rather small as estimated by a DFT calculation $\left(E_{\mathrm{cub}}-E_{\mathrm{tri}}=2.3 \mathrm{~kJ} \cdot \mathrm{mol}^{-1}\right)$. The disordered structural 


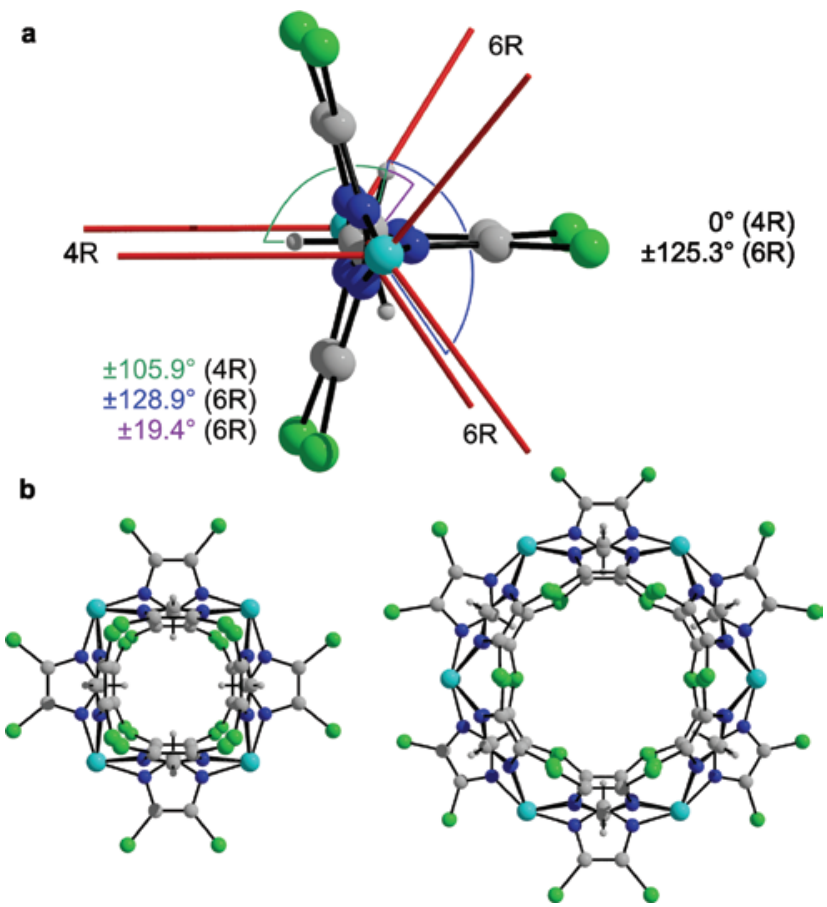

Fig. 6: (a) Illustration of the statistical disorder of the two unique dcim linkers over three principal orientations in the unique $\mathrm{Zn} \cdots \mathrm{Zn}$ edge of the cubic SOD-type material with the linker twist angles indicated. (b) Unique four- and six-membered rings with linker disorder. Color code: cyan, $\mathrm{Zn}$; blue, $\mathrm{N}$; gray, $\mathrm{C}$ and $\mathrm{H}$; green, $\mathrm{Cl}$. Reproduced from Ref. [20] with permission from the Royal Society of Chemistry.

model was subsequently confirmed by Rietveld analysis with higher-quality powder XRD data recorded from an as-made (THF-containing) sample. Activation of the as-made cubic material was found to occur with retention of framework symmetry $(\operatorname{Im} \overline{3} m)$, yet with significant loss of crystallinity.

As structural analysis has revealed, structural differences of the prepared trigonal (ordered) and cubic (disordered) SOD-type materials are more related to characteristics of real as opposed to ideal crystal structures, both SOD-type materials should not be considered as true conformational polymorphs. However, their experimental realization is clearly in line with our theoretically predicted potential static conformational variety of the $\left[\mathrm{Zn}(\text { dcim })_{2}\right]$-SOD framework.

\section{Characterization of the trigonal and cubic $\left[\mathrm{Zn}(\mathrm{dcim})_{2}\right]-S O D$ materials}

Comparative characterization of the trigonal and cubic SOD-type materials was of interest because it enabled an investigation of the influence real structural phenomena can have on materials properties, in this case static conformational disorder. Defects in ZIFs have scarcely been addressed experimentally so far [40-42].

Argon adsorption/desorption isotherms measured at cryogenic temperature from activated samples of the trigonal and cubic SOD-type materials exhibit clear differences (Figure 7).

In the case of the trigonal material, the steep argon uptake in the low-pressure regime, typical for microporous materials, is a two-step process, as is commonly observed in flexible ZIF frameworks. Here, the second step is likely due to some small reorientational movements of the dcim linkers upon reaching a certain threshold pressure to allow accommodation of additional argon atoms. Such guest-induced displacive framework transitions may (e.g. ZIF-7 [33, 34, 43]) or may not (e.g. ZIF-8 [30, 31] and ZIF-4 [44]) occur with a change of symmetry. In contrast, a similar step is not seen in the low-pressure region in case of the cubic material, demonstrating that static orientational linker disorder can affect framework dynamics.

At pressures beyond the steep uptake, a clear (first) plateau is reached in case of the trigonal material, while in case of the cubic material uptake smoothly continues and a shallow hysteresis appears between the adsorption and desorption branches. The latter uptake behavior provides evidence for the presence of mesopores with a broad size distribution as internal volume defects, which is not an unexpected finding for a disordered material. However, the hysteresis loop seen at large pressures in

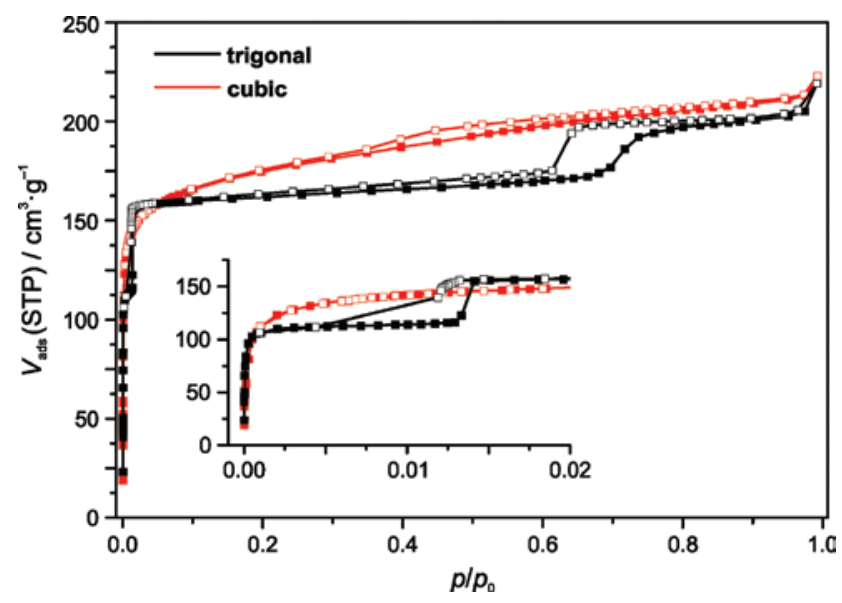

Fig. 7: Argon sorption isotherms of the trigonal and cubic SOD-type materials at $87 \mathrm{~K}$ with adsorption (closed squares) and desorption (open squares) branches. The inset shows an expansion of the low pressure region. Reproduced from Ref. [20] with permission from the Royal Society of Chemistry. 
case of the apparently (according to XRD) well crystalline trigonal material is unexpected. The shape of the hysteresis is indicative for the presence of mesopores with a quite narrow size distribution centered at about $6 \mathrm{~nm}$. The origin of the hysteresis is not yet well understood, but it may be connected with the crystallization mechanism (see below).

\section{Discovery of intermediates of the trigonal and cubic $\left[\mathrm{Zn}\left(\mathrm{dcim}_{2}\right]\right.$-SOD materials}

Investigations of the influence of various solvents on phase formation in the $\left[\mathrm{Zn}(\mathrm{dcim})_{2}\right]$ system indicated the importance of the correct choice of solvent for the crystallization of the trigonal SOD-III polymorph, as it could only be obtained in DMF under solvothermal conditions. Powder XRD patterns recorded at two different time intervals revealed that the crystallization takes place via the transient formation of a low-symmetry variant of the cubic SOD-type material (Figure 8), suggesting a solutionmediated reconstruction process as a possible mechanism of SOD-III crystal growth. Two experimental findings are in support of such a mechanism: the observed SOD-III crystal morphologies, ranging from irregular to rhombicdodecahedron-like agglomerates, and the gas-uptake properties, which indicate the presence of mesopores. However, confirmation of this hypothesis needs further careful investigations.

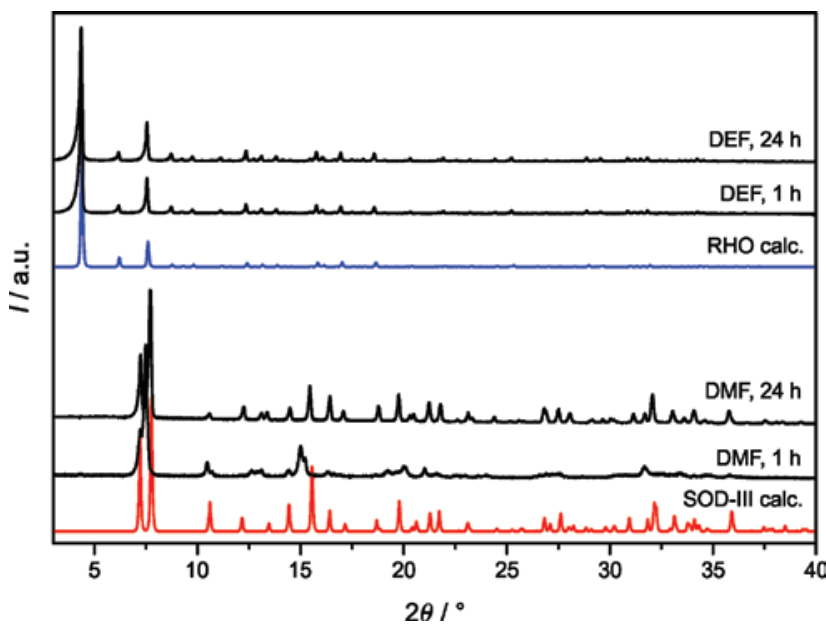

Fig. 8: Typical powder XRD patterns of the products obtained in dimethylformamide (DMF) and diethylformamide (DEF) after 1 and $24 \mathrm{~h}$. Also shown are simulated powder XRD patterns for the trigonal SOD-III and RHO polymorphs.
Experiments with other common solvents (e.g. DEF, various alcohols) yielded exclusively the RHO-type polymorph (in case of DEF) or the RHO-type, low-symmetry variants of the cubic SOD-type, and lcs-type materials in relative amounts that depended on the reaction conditions (type of alcohol, temperature, time). The less commonly used THF was found to result exclusively in the crystallization of the cubic SOD-type material. This took place even at room temperature and after prolonged times of heating (up to 7 days), indicating that a transformation into the trigonal SOD-III polymorph, as it was observed in DMF, takes place at a very slow rate or not at all. According to our synthetic work, good structure-directing agents for the RHO-type, trigonal and cubic SOD-type materials are DEF, DMF and THF, respectively. The solvents also serve as good stabilizing agents for the respective frameworks, as transformations to the stable lcs-type polymorph were not observed to occur under the reaction conditions considered.

As DMF and THF were found to direct crystallization of the trigonal and cubic SOD-type material, respectively, it seemed natural to investigate phase formation in DMF/ THF solvent mixtures. The powder XRD patterns recorded from the obtained products (Figure 9) indicate that there exists a continuous series of intermediate SOD-type frameworks with the trigonal and cubic frameworks as the end members (in line with additionally taken SEM images).

It is our hypothesis that the intermediate materials consist, as the cubic end-member material, of domains of
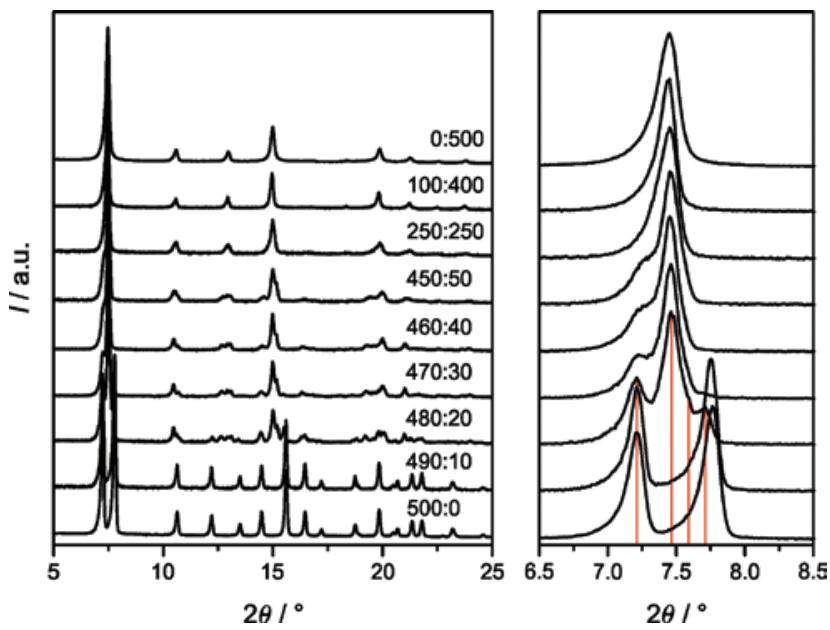

Fig. 9: Powder XRD patterns of the products obtained in various $\mathrm{DMF} / \mathrm{THF}$ mixtures with the molar DMF/THF ratios indicated. The enlargement on the right-hand side clearly reveals the features of the strongest reflections at about $7.5^{\circ} 2 \theta$; the red bars mark the positions of at least four reflections (three peaks and one shoulder) in the 480:20 pattern. Reproduced from Ref. [20] with permission from the Royal Society of Chemistry. 
the SOD-III conformer, differing in the degree of structural deformation, domain size and domain interface structure. It is remarkable that already a very small amount of THF in DMF (ratio DMF/THF = 480:20) hindered crystallization of the pure, undistorted trigonal SOD-III conformer. The series of discovered intermediates also indicates that conformations in the $\left[\mathrm{Zn}\left(\mathrm{dcim}_{2}\right]\right.$-SOD framework may be controlled by careful choice of synthetic conditions, possibly allowing access to other predicted SOD-type structures with different properties.

\section{Investigations on [Zn(dcim) $\left.{ }_{2}\right]-$ RHO/ SOD phase selection via coordination modulation}

In our previous work, we had discovered that 1-methylimidazole (1-mim) can apparently be used as an additive to synthesis mixtures for controlling phase selection during competitive crystallization of the kinetically favored RHOtype polymorph and a less favored SOD-type material (a low-symmetry variant of cubic [Zn(dcim) $)_{2}$-SOD) [22].
We hypothesized that 1-mim acts as a monodentate ligand in competition to the bidentate dcim linker in the coordination equilibria during the self-assembly processes and, with increasing relative amount, eventually suppresses the prior formation of the favored RHO-type polymorph, resulting in the selective formation of the SOD-type material. In order to verify that hypothesis we performed in-situ time-resolved XRD experiments using monochromatic high-energy synchrotron radiation and a newly developed laboratory-scale reactor (see Experimental section). It must be noted that the in-situ experiments were performed with comparatively dilute solutions, corresponding to the synthesis protocols that had originally been designed to produce nanocrystalline material [22]. Therefore, quality of the recorded XRD patterns in particular towards larger scattering angles ( $q$ values) and severe RHO/SOD peak overlapping do not enable any quantitative analysis [45-48]. Nonetheless, valuable qualitative information could be provided.

Figure 10 shows time-dependent XRD patterns recorded at $50{ }^{\circ} \mathrm{C}$ from reaction mixtures of composition $\mathrm{Zn}\left(\mathrm{NO}_{3}\right)_{2} \cdot 6 \mathrm{H}_{2} \mathrm{O} / \mathrm{Hdcim} / 1$-mim/1-propanol = 1:4: $x: 1000$ with $0 \leq x \leq 4$. For the non-modulated reaction $(x=0)$ rapid formation of the RHO-type polymorph is seen after
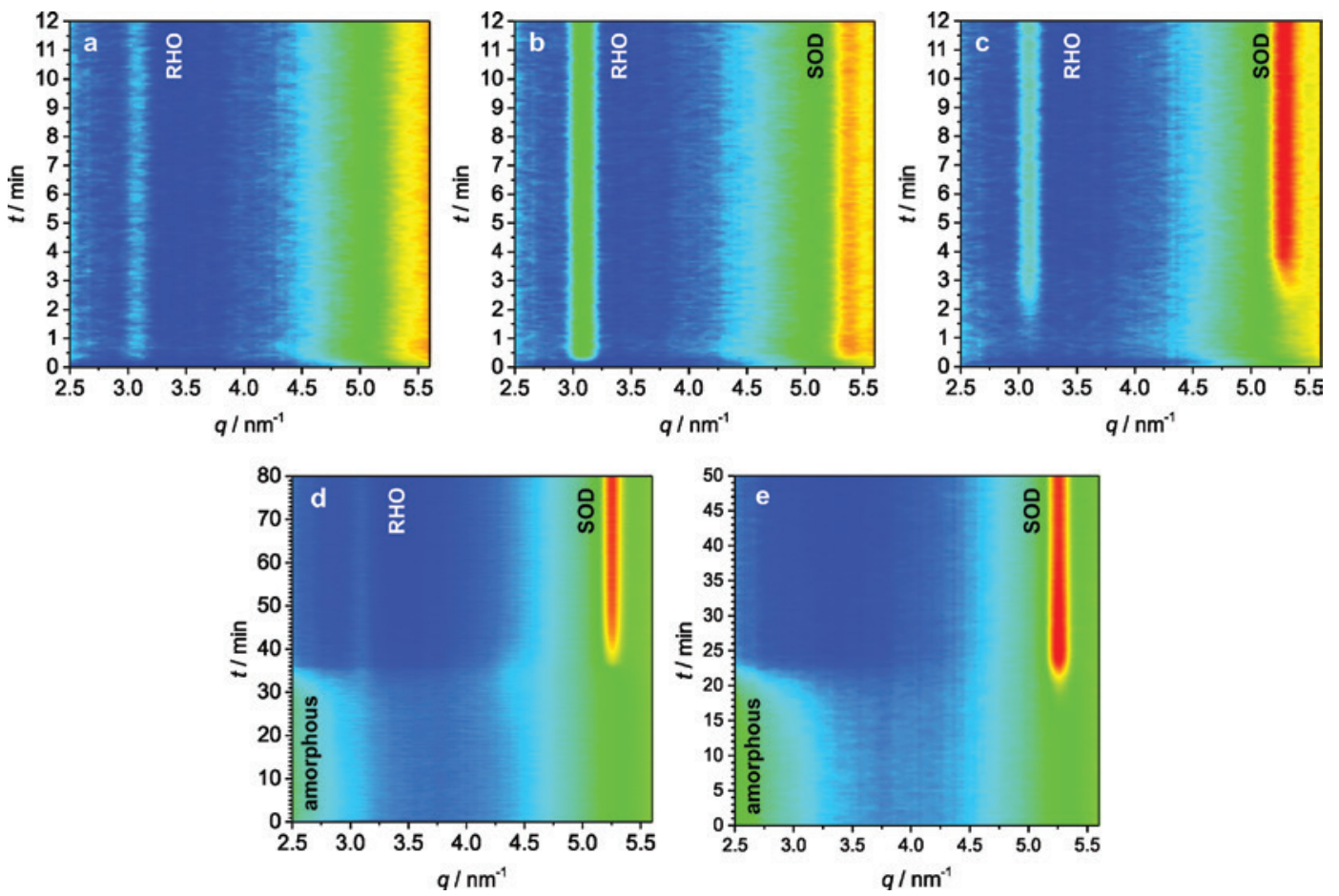

Fig. 10: Contour plots of the XRD patterns recorded in situ for the solutions of composition Zn/Hdcim/1-mim/1-propanol=1:4:x:1000 with $x=0$ (a), 1 (b), 2 (c), 3 (d) and 4 (e). The phases from which the Bragg peaks (RHO and SOD) and broad scattering features (amorphous phase) originate are indicated. XRD data was recorded in intervals of $5 \mathrm{~s}$ for $x=0,1,2$, and $25 \mathrm{~s}$ for $x=3,4$, respectively. In the cases of $x=1$ and 2 , the peak at $5.3 \mathrm{~nm}^{-1}$, indicated with SOD, actually contains a small contribution form a RHO peak due to strong peak overlapping. 
ca. $5 \mathrm{~s}$. It is known from ex-situ experiments that this phase transforms into a variant of the cubic SOD-type material after about $1 \mathrm{~h}$ (at room temperature) [22]. At the smallest amount of added 1-mim $(x=1)$, crystallization begins at about the same time ( $c a .10 \mathrm{~s}$ ), but now the RHO-type and cubic SOD-type materials form almost simultaneously. Compared with the non-modulated reaction, the RHO-type polymorph is formed in higher yield, as can be inferred from the increased intensity of the 110 Bragg peak. This observation may be explained if it is assumed that 1-mim acts, at this low amount, as a base to deprotonate the Hdcim linker molecules (deprotonation modulation), thereby shifting the gross equilibrium to the product side [49]. The reaction kinetics is slowed down with further addition of 1-mim $(x=2)$ and the RHOtype polymorph forms after $c a .60 \mathrm{~s}$ in lower yield, followed by the formation of the SOD-type material after 150 s. At $x=3$, the RHO-type polymorph still forms in very low yield, but its first appearance is hardly visible in the broad scattering feature that is now seen at small $q$ values. This feature indicates the transient formation of an amorphous phase. The SOD-type material appears after $37 \mathrm{~min}$, while the amorphous phase disappears. At the largest amount of added 1-mim $(x=4)$ the RHO-type polymorph does not appear anymore. Only the amorphous phase forms transiently, followed by the SOD-type material after $21 \mathrm{~min}$.

Suppression of the formation of the favored polymorph through addition of the modulator has thus been experimentally verified and may be attributed to the masking of the active "monomer" (which is probably some distribution of oligomers [14]). This masking decreases the active concentration below the critical concentration for nucleation of the favored (more soluble) material, but still keeps it above the critical concentration for nucleation of the less favored (less soluble) material. However, the fact that formation of the less favored SOD-type material in the reactions with $x=3$ and 4 took place at an earlier time with the larger amount of 1-mim contradicts the trend suggested by the simple coordination modulation mechanism. On the other hand, it should be considered that many mechanistic details still remain unclear, e.g. the possible involvement of the amorphous and RHO-type phases (the latter is only observed at $x=3$ ) and the true chemical nature of the monomer.

Coordination modulation, also known as coordination buffering, has been previously proposed as an explanation for polymorph (supramolecular isomer) selection in coordination polymers by other researchers [50-52], but the true function of the modulators still remained unclear, because mechanistic studies are missing. Our time-resolved in-situ investigations provide for the first time some insight.

\section{Investigations on rapid [Zn(dcim) $\left.)_{2}\right]$ RHO formation}

As reported previously [22], the formation of nanocrystals of the RHO-type polymorph in comparatively dilute 1-propanolic solutions at room temperature is a very fast process, which runs on a time-scale ranging from seconds to a few minutes in the absence of any modulator (also, see the in-situ XRD studies above). This reaction was studied in detail using in-situ static and dynamic light scattering (SLS/DLS) as well as stopped-flow synchrotron small-angle and wide-angle X-ray scattering (SAXS/ WAXS) methods with a resolution on time- and lengthscale suitable to gain information about the entire homogenous crystallization process.

Time-resolved SLS experiments performed on solutions with varying zinc/linker ratios at fixed zinc concentrations revealed that the nucleation kinetics becomes faster with increasing amount of linker, resulting in final nanocrystals with decreasing size. The SLS data enabled us to establish the same power-law relation between particles size (radius of gyration) and mass for all investigated reactions, which suggests that particle growth occurs by a monomer-addition mechanism throughout the entire regime of linker concentrations with the monomers corresponding to not yet identified small entities. Furthermore, from a combined SLS/DLS experiment the ratio of the radius of gyration and the hydrodynamic radius, which depends on the particle morphology, could be determined as a function of time. The evolution of that ratio with time indicated that the particles kept the same compact and isometric shape during their growth.

While in-house SLS was limited to the monitoring of particles larger than approximately $60 \mathrm{~nm}$ in diameter as lower limit of size resolution, the combined SAXS/ WAXS experiments at beamline ID02 (ESRF) on selected reactions provided also insight into nucleation and early growth events. In the scattering patterns the evolution of four species could be followed: small entities (termed clusters), bigger entities (termed particles), crystallites and aggregates. For a reaction mixture of composition $\mathrm{Zn}\left(\mathrm{NO}_{3}\right)_{2} \cdot 6 \mathrm{H}_{2} \mathrm{O} / \mathrm{Hdcim} / 1$-propanol $=1: 4: 2000$, the development of size and mass for the clusters and particles, as determined by model-independent analysis of the SAXS data, and the extent of crystallization, as determined from 
the intensities of the 110 reflection of the RHO-type polymorph in the WAXS data, are shown in Figures 11 and 12, respectively.

The clusters were seen already in the second measurement after $0.6 \mathrm{~s}$, while the first particles formed only after $15 \mathrm{~s}$. Our data provide strong evidence that these first entities were amorphous, as the 110 Bragg peak was detected not before $\mathrm{ca} .70 \mathrm{~s}$, corresponding to an experimental extent of crystallization of $c a$. 0.3 , and an estimated induction time for the nucleation of RHO-type crystallites of $c a$. $35 \mathrm{~s}$. At the end of the induction time, when the clusters apparently began to disappear, the particles had grown already to more than three quarters of their final size (diameter of $c a .180 \mathrm{~nm}$ ). As the rate of crystal nucleation was estimated to be larger than the rate of crystal growth, we hypothesize that crystallization is more an intra-particle reconstruction than a dissolution-recrystallization process. Interestingly, analysis of the SAXS data yielded different power-law relations between size and scattered

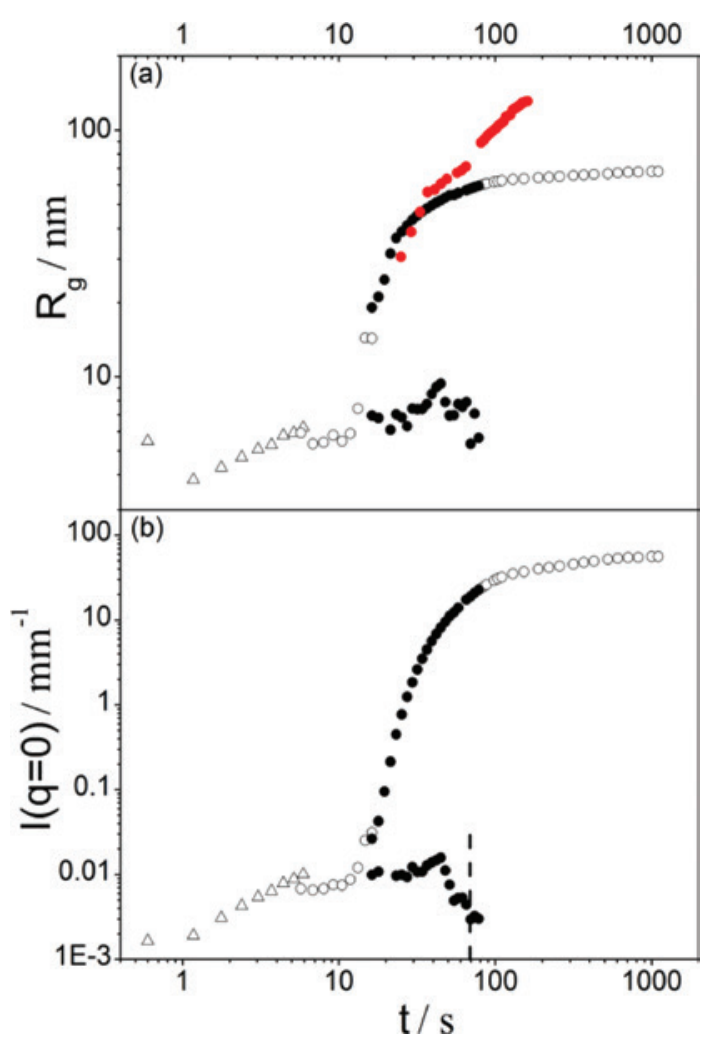

Fig. 11: Evolution with time of (a) radius of gyration and (b) scattering intensity at $q=0$ for clusters and particles, as extracted from in-situ SAXS data (black and white symbols), and for aggregates, as extracted from in-situ SLS data (red symbols), for a solution of composition $\mathrm{Zn} / \mathrm{dcim} / 1$-propanol=1:4:2000. The dashed vertical line indicates the first appearance of the 110 Bragg peak of the RHO-type polymorph. Reprinted from Ref. [21]. Copyright (C) 2016 American Chemical Society.

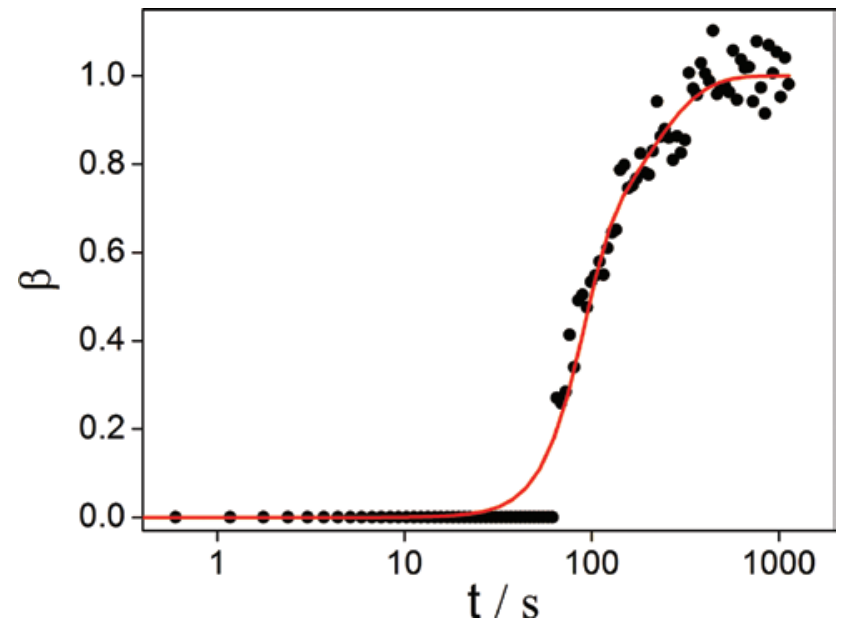

Fig. 12: Extent of crystallization, determined from the 110 Bragg peak of the RHO-type polymorph in the WAXS region, as function of time. The red line was obtained by fitting the data points with the Gualtieri formula [53]. Reprinted from Ref. [21]. Copyright (c) 2016 American Chemical Society.

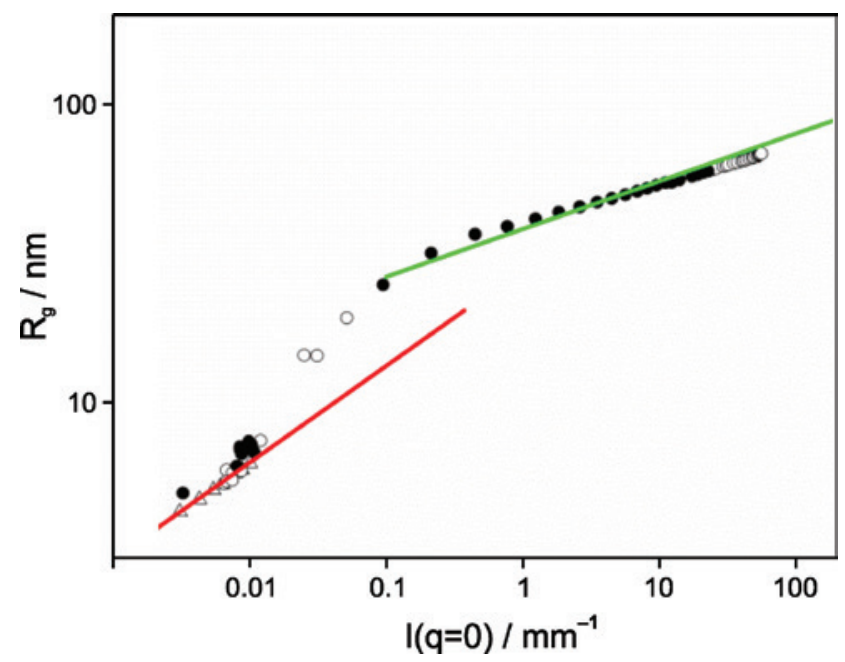

Fig. 13: Correlation between the radius of gyration and the scattered intensity extrapolated to $q=0$ established from in-situ SAXS experiments for a solution of composition $\mathrm{Zn} / \mathrm{dcim} / 1$ propanol $=1: 4: 2000$. The colored lines correspond to $\alpha$ values of $1 / 3$ (red) and $1 / 6$ (green) in the power law relation $\mathrm{R}_{\mathrm{g}} \propto I(q=0)^{\alpha}$. Reprinted from Ref. [21]. Copyright () 2016 American Chemical Society.

intensity at $q=0$ for clusters and particles (Figure 13), suggesting a coalescence mechanism for growth of clusters (involving clusters and smaller units), in contrast to the monomer-addition growth of particles with clusters and/ or smaller units as the possible monomers.

On the basis of the above analysis we propose a mechanism of $\left[\mathrm{Zn}(\mathrm{dcim})_{2}\right]-\mathrm{RHO}$ formation that is illustrated in Figure 14. The mechanistic scheme starts with "Zn-dcim 


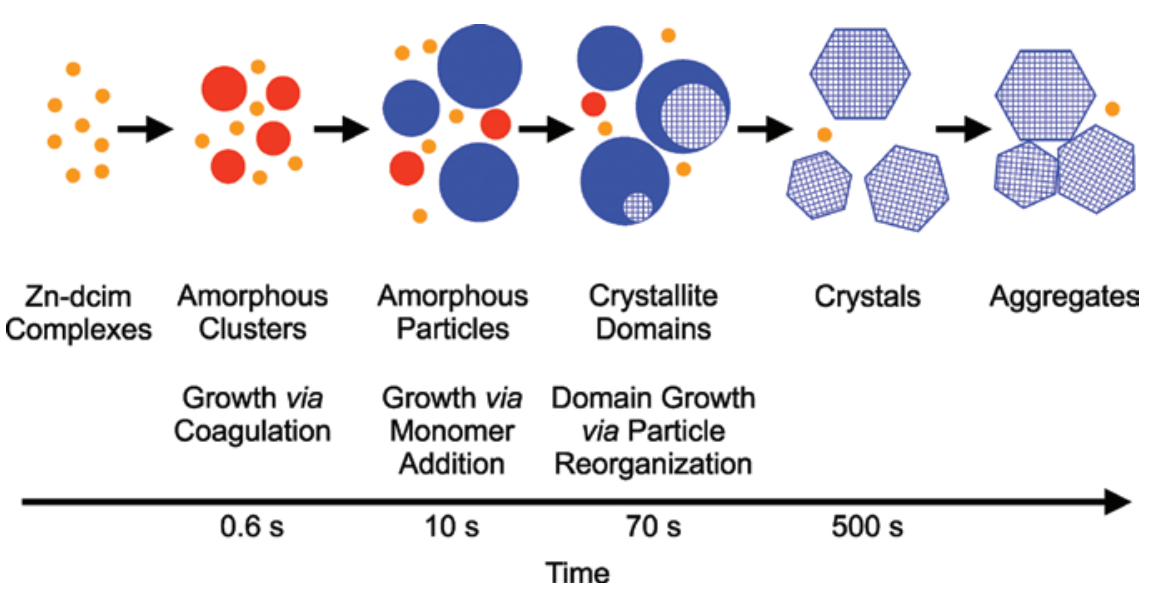

Fig. 14: A simplistic scheme outlining the proposed steps and intermediates occurring during the formation of the RHO-type polymorph. The times given correspond to the in-situ experiment with the composition Zn/Hdcim/1-propanol=1:4:2000. Reprinted from Ref. [21]. Copyright @ 2016 American Chemical Society.

complexes", meaning multinuclear species (oligomers) that probably form first during the pre-nucleation stage via complex formation, complex deprotonation and ligand exchanges reactions. Previous in-situ ESI-MS investigations on ZIF-8 formation have demonstrated the transient formation of such oligomers containing up to seven $\mathrm{Zn}$ ions [14]. The mechanistic scheme is compatible with the knowledge gathered so far for ZIF-8 formation $[14-17,54,55]$ and may thus be more generally valid for ZIF formation in comparatively dilute solutions independent from topology. Concerning clusters and particles, our investigations above have added significant new details to the mechanism. However, the mechanism differs partly from that observed for the formation of dense $\left[\mathrm{Zn}(\mathrm{im})_{2}\right]-$ zni in similar dilute solution, where a particle growth by monomer addition with the particle as the monomer occurs $[1,56]$.

\section{Conclusions}

Theoretical studies on the zeolitic imidazolate framework (ZIF) system $\left[\mathrm{Zn}(\mathrm{dcim})_{2}\right](\mathrm{dcim}=4,5$-dichloroimidazolate) predict that besides a number of topological polymorphs, there potentially exists an infinite number of conformational [ $\left.\mathrm{Zn}(\mathrm{dcim})_{2}\right]$-SOD polymorphs with the same underlying sodalite topology but distinct linker orientations (including periodic as well as non-periodic and defective frameworks) that exhibit comparatively small energetic differences. In line with those predictions of a great static conformational variety of the [Zn(dcim) $)_{2}$-SOD framework, we have so far been able to experimentally realize two SOD-type materials: the first (ordered) trigonal SOD-type material is identical with the most stable of the predicted conformational polymorphs, while the second cubic SOD-type material exhibits orientational disorder of the linkers. The disorder is most likely not random, rather we hypothesize that the cubic crystals consist of nanoscopic domains of the trigonal polymorph deformed to a cubic metric. In addition, a complete series of intermediate frameworks with the trigonal and cubic structures as the end members has been synthesized. Argon physisorption measurements have revealed that the static orientational disorder of the linkers affects the dynamics of the $\left[\mathrm{Zn}(\mathrm{dcim})_{2}\right]-\mathrm{SOD}$ framework. The three so far experimentally realized topological [ $\left.\mathrm{Zn}(\mathrm{dcim})_{2}\right]$ polymorphs with ordered frameworks (RHO, SOD, lcs) are amongst the most stable predicted phases in the $\left[\mathrm{Zn}\left(\mathrm{dcim}_{2}\right)_{2}\right.$ system.

In-situ XRD studies have demonstrated for the first time that addition of a monodentate ligand (coordination modulator) can completely suppress the crystallization of a kinetically favored (more soluble) RHO-type polymorph under conditions of competitive RHO-/SOD-type phase formation, resulting in the exclusive crystallization of the less favored (less soluble) SOD-type polymorph. In-situ light and SAXS/WAXS studies have provided insight into the entire rapid homogeneous crystallization process of the RHO-type polymorph: small entities (termed clusters) first from via a coagulation reaction, followed by amorphous particles that grow via a monomer-addition reaction with the clusters or smaller units as the monomers. The particles transform into the periodic RHO-type structure probably via intra-particle nucleation and subsequent particle reorganization. 


\section{Experimental}

Details concerning structural derivations and DFT calculations as well as experimental work can be found elsewhere [20, 21]. The in-situ XRD experiments on RHO/SOD phase selection via coordination modulation, which are reported for the first time in the present contribution, are described in the following.

Time-resolved in-situ synchrotron angular-dispersive XRD experiments were performed at the side station P07b of the High Energy Materials Science beamline P07 of storage ring PETRA III at photon light source DESY (Hamburg, Germany) using monochromatic radiation with energy of $87.1 \mathrm{keV}$ (wavelengths: $0.14235 \AA$ ). . 2D patterns were recorded using a Perkin Elmer XRD 1621 digital X-ray flat panel detector. The reactions were carried out in a custom-built reactor that was developed at CAU Kiel in cooperation with the beamline staff of beamline P08, PETRA III, DESY and shall be described in detail in a separate publication. The reactor enables the in-situ monitoring of reactions under solvothermal conditions via synchrotron-based techniques. The main part of the reactor consists of an aluminum casing surrounded by copper wires, which is aligned in transmission geometry on the beamline. Duran (C)-borosilicate glass tubes with a maximum volume of $\sim 5 \mathrm{~mL}$ were used as reaction vessels. The temperature was monitored throughout the entire reaction with a PTFE-coated thermocouple located inside the reaction vessel. Through a combination of resistive heating and direct cooling via compressed air was the temperature inside the glass vessel precisely controlled within $\pm 0.5^{\circ} \mathrm{C}$ of the set reaction temperature. The glass vessel was closed via a cap with integrated tubes, which were connected to syringe pumps. A 1-propanolic $\mathrm{Zn}\left(\mathrm{NO}_{3}\right)_{2} \cdot 6 \mathrm{H}_{2} \mathrm{O}$ solution was first filled into the glass tube and pre-heated in the reactor to $50{ }^{\circ} \mathrm{C}$ for $5 \mathrm{~min}$. Then, a 1-propanolic Hdcim solution containing the desired amount of 1-mim was injected via the pumping system into the stirred zinc salt solution with a flow rate of $10 \mathrm{~mL} \cdot \mathrm{min}^{-1}$. The total volume of the combined solutions was $3 \mathrm{~mL}$. The pumping and heating systems of the reactor were synchronized with the XRD data acquisition system. 2D data were azimuthally integrated using the Fit2D program [57] to obtain a 1D profile.

Acknowledgments: Provision of beamtime at beamline P07 by DESY and HZG is gratefully acknowledged. We thank Norbert Schell and Uta Rütt for support at the beamline during the in-situ experiments. Financial support by the Deutsche Forschungsgemeinschaft (DFG) within the framework of the priority program 1415 ("Crystalline NonEquilibrium Phases”) is gratefully acknowledged.

\section{References}

[1] C. A. Schröder, S. Saha, K. Huber, S. Leoni, M. Wiebcke, Metastable metal imidazolates: development of targeted syntheses by combining experimental and theoretical investigations of the formation mechanisms. Z. Kristallogr. 2014, 229, 807.

[2] J.-P. Zhang, Y.-B. Zhang, J.-B. Lin, X.-M. Chen, Metal azolate frameworks: from crystal engineering to functional materials. Chem. Rev. 2012, 112, 1001.
[3] A. Phan, C. J. Doonan, F. J. Uribe-Romo, C. B. Knobler, M. O’Keeffe, O. M. Yaghi, Synthesis, structure, and carbon dioxide capture properties of zeolitic imidazolate frameworks. Acc. Chem. Res. 2010, 43, 58.

[4] J.-F. Yao, H.-T. Wang, Zeolitic imidazolate framework composite membranes and thin films: synthesis and applications. Chem. Soc. Rev. 2014, 43, 4470.

[5] D. Farrusseng, S. Aguado, C. Pinel, Metal-organic frameworks: opportunities for catalysis. Angew. Chem. Int. Ed. 2009, 48, 7502.

[6] W. Cai, C.-C. Chu, G. Liu, Y.-X. Wáng, Metal-organic frameworkbased nanomedicine platforms for drug delivery and molecular imaging. Small 2015, 11, 4806.

[7] B.-L. Chen, Z.-X. Yang, Y.-Q. Zhu, Y. D. Xia, Zeolitic imidazolate framework materials: recent progress in synthesis and applications. J. Mater. Chem A 2014, 2, 16811.

[8] N. Stock, S. Biswas, Synthesis of metal-organic frameworks (MOFs), Routes to various MOF topologies, morphologies, and composites. Chem. Soc. Rev. 2012, 112, 933.

[9] M. G. Goesten, F. Kapteijn, J. Gascon, Fascinating chemistry or frustrating unpredictability: observations in crystal engineering of metal-organic frameworks. CrystEngComm 2013, 15, 9249.

[10] I. A. Baburin, S. Leoni, The energy landscape of zeolitic imidazolate frameworks (ZIFs): towards quantifying the presence of substituents on the imidazole ring. J. Mater. Chem. 2012, 22, 10152.

[11] R. Galvelis, B. Slater, R. Chaudret, B. Creton, C. Nieto-Draghi, C. Mellot-Draznieks, Impact of functionalized linkers on the energy landscape of ZIFs. CrystEngComm 2013, 15, 9603.

[12] J. A. Gee, D. S. Sholl, Characterization of the thermodynamic stability of solvated metal-organic polymorphs using molecular simulations. J. Phys. Chem. C 2013, 117, 20636.

[13] L. Bouessel du Bourg, A. U. Ortiz, A. Boutin, F.-X. Coudert, Thermal and mechanical stability of zeolitic imidazolate framework polymorphs. APL Mater. 2014, 2, 124110.

[14] I. H. Lim, W. Schrader, F. Schüth: Insights into the molecular assembly of zeolitic imidazolate frameworks by ESI-MS. Chem. Mater. 2015, 27, 3088.

[15] J. Cravillon, R. Nayuk, S. Springer, A. Feldhoff, K. Huber, M. Wiebcke, Controlling zeolitic imidazolate framework nano- and microcrystal formation: insight into crystal growth by time-resolved in situ static light scattering. Chem. Mater. 2011, 23, 2130.

[16] J. P. Patterson, P. Abellan, M. S. Denny Jr., C. Park, N. D. Browning, S. M. Cohen, J. E. Evans, N. C. Gianneschi, Observing the growth of metal-organic frameworks by in situ liguid cell transmission electron microscopy. J. Am. Chem. Soc. 2015, 137, 7322.

[17] J. Cravillon, C. A. Schröder, R. Nayuk, J. Gummel, K. Huber, M. Wiebcke, Fast nucleation and growth of ZIF-8 nanocrystals monitored by time-resolved in situ small-angle and wide-angle X-Ray scattering. Angew. Chem. Int. Ed. 2011, 50, 8067.

[18] Z.-X. Low, J.-F. Yao, Q. Liu, M. He, Z.-Y. Wang, A. K. Suresh, J. Bellare, H.-T. Wang, Crystal transformation in a Zeolitic-Imidazolate framework. Cryst. Growth Des. 2014, 14, 6589.

[19] K. Self, M. Telfer, H. F. Greer, W. Z. Zhou, Revered crystal growth of RHO zeolitic imidazolate framework (ZIF). Chem.- Eur. J. 2015, 21, 19090.

[20] S. Springer, I. A. Baburin, T. Heinemeyer, J. G. Schiffmann, L. van Wüllen, S. Leoni, M. Wiebcke, A zeolitic imidazolate 
framework with conformational variety: conformational polymorphs versus frameworks with static conformational disorder. CrystEngComm 2016, 18, 2477.

[21] S. Saha, S. Springer, M. E. Schweinefuß, D. Pontoni, M. Wiebcke, K. Huber, Insight into fast nucleation and growth of zeolitic imidazolate framework-71 by in situ time-resolved light and X-ray scattering. Cryst. Growth Des. 2016, 16, 2002.

[22] M. E. Schweinefuß, S. Springer, I. A. Baburin, T. Hikov, K. Huber, S. Leoni, M. Wiebcke, Zeolitic imidazolate framework-71 nanocrystals and a novel SOD-type polymorph: solution mediated phase transformations, phase selection via coordination modulation and a density functional theory derived energy landscape. Dalton Trans. 2014, 43, 3528.

[23] R. Sabatini, T. Gorni, S. de Gironcoli, Nonlocal van der Waals density functional made simple and efficient. Phys. Rev. B: Condens. Matter 2013, 87, 041108.

[24] International Zeolite Association, Database of Zeolite structures. http://www.iza-structure.org/databases.

[25] R. Banerjee, A. Phan, B. Wang, C. Knobler, H. Furukawa, M. O'Keeffe, O. M. Yaghi, High-throughput synthesis of zeolitic imidazolate frameworks and application to $\mathrm{CO}_{2}$ capture. Science 2008, 319, 939.

[26] W. Depmeier, The sodalite family - a simple but versatile framework structure. Rev. Mineral. Geochem. 2005, 57, 203.

[27] X.-C. Huang, Y.-Y. Lin, J.-P. Zhang, X.-M. Chen, Ligand-directed strategy for zeolite-type metal-organic frameworks: zinc(II) imidazolates with unusual zeolitic topologies. Angew. Chem. Int. Ed. 2006, 45, 1557.

[28] K. S. Park, Z. Ni, A. P. Côté, J. Y. Choi, R. Huang, F. J. UribeRomo, H. K. Chae, M. O'Keeffe, O. M. Yaghi, Exceptional chemical and thermal stability of zeolitic imidazolate frameworks. Proc. Nat. Acad. Sci. USA 2006, 103, 10186.

[29] S. A. Moggach, T. D. Bennett, A. K. Cheetham, The effect of pressure on ZIF-8: Increasing pore size with pressure and the formation of a high-pressure Phase at $1.47 \mathrm{GPa}$. Angew. Chem. Int. Ed. 2009, 48, 7087.

[30] D. Fairen-Jimenez, S. A. Moggach, M. T. Wharmby, P. A. Wright, S. Parsons, T. Düren, Opening the gate: framework fexibility in ZIF-8 explored by experiments and simulations. J. Am. Chem. Soc. 2011, 133, 8900.

[31] J.-P. Zhang, A.-X. Zhu, X.-M. Chen, Single-crystal X-ray diffraction and Raman spectroscopy studies of the isobaric $\mathrm{N}_{2}$ adsorption in SOD-type metal-organic zeolites. Chem. Commun. 2012, 48, 11395.

[32] X-C. Huang, J.-P. Zhang, X.-M. Chen, $\left[\mathrm{Zn}(\mathrm{bim})_{2}\right] \cdot\left(\mathrm{H}_{2} \mathrm{O}\right)_{1.66}: \mathrm{A}$ metal-organic open-framework with sodalite topology. Chin. Sci. Bull. 2003, 48, 1531.

[33] P. Zhao, G. I. Lampronti, G. O. Lloyd, M. T. Wharmby, S. Facq, A. K. Cheetham, S.Redfern, phase transitions in zeolitic imidazolate framework 7: the importance of framework flexibility and guest-induced instability. Chem. Mater. 2014, 26, 1767.

[34] Y. Du, B. Wooler, M. Nines, P. Kortunov, C. S. Pauer, J. Zengel, S. C. Weston, P. I. Ravikovitch, New high- and low-temperature phase changes of ZIF-7: elucidation and prediction of the thermodynamics of transitions. J. Am. Chem. Soc. 2015, 137, 13603.

[35] K. Knorr, C. M. Braunbarth, G. van der Goor, P. Behrens, C. Griewatsch, W. Depmeier, High-pressure study on dioxolane silica sodalite $\left(\mathrm{C}_{3} \mathrm{H}_{6} \mathrm{O}_{2}\right)_{2}\left[\mathrm{Si}_{12} \mathrm{O}_{24}\right]-$ neutron and $\mathrm{X}$-ray powder diffraction experiments. Solid State Commun. 2000, 503, 114.
[36] R. S. P. King, S. E. Dann, M. R. J. Elsegood, P. F. Kelly, R. J. Mortimer, The synthesis, full characterisation and utilisation of template-free silica sodalite, a novel polymorph of silica. Chem.- Eur. J. 2009, 15, 5441.

[37] F.-X. Coudert, Responsive metal-organic frameworks and framework materials: under pressure, taking the heat, in the spotlight, with friends. Chem. Mater. 2015, 27, 1905.

[38] I. A. Baburin, S. Leoni, Modelling polymorphs of metal-organic frameworks: a systematic study of diamondoid zinc imidazolates. CrystEngComm 2010, 12, 2809.

[39] A.-X. Zhu, R.-B. Lin, X.-L. Qi, Y. Liu, Y.-Y. Lin, J.-P. Zhang, X.-M. Chen, Zeolitic metal azolate frameworks (MAFs) from $\mathrm{ZnO} /$ $\mathrm{Zn}(\mathrm{OH})_{2}$ and monoalkyl-substituted imidazoles and 1,2,4-triazoles: Efficient syntheses and properties. Microporous Mesoporous Mater. 2012, 157, 42.

[40] D. S. Sholl, R. P. Lively, Defects in metal-organic frameworks: challenge or opportunity? J. Phys. Chem. Lett. 2015, 6, 3437.

[41] C. Zhang, C. Han, D. S. Sholl, J. R. Schmidt, Computational characterization of defects in metal-organic frameworks: spontaneous and water-induced point defects in ZIF-8. J. Phys. Chem. Lett. 2016, 7, 459.

[42] Z. Fang, B. Bueken, D. E. de Vos, R. A. Fischer, Defect-engineered metal-organic frameworks. Angew. Chem. Int. Ed. 2015, 54, 7234.

[43] S. Aguado, G. Bergeret, M. P. Titus, V. Moizan, C. Nieto-Draghi, N. Bats, D. Farrusseng, Guest-induced gate-opening of a zeolitic imidazolate framework. New J. Chem. 2011, 35, 546.

[44] M. T. Wharmby, S. Henke, T. D. Bennett, S. R. Bajpe, I. Schwedler, S. P. Thompson, F. Gozzo, P. Simoncic, C. Mellot-Draznieks, H. Tao, Y. Yue, A. K. Cheetha, Extreme flexibility in a zeolitic imidazolate framework: porous to dense phase transition in desolvated ZIF-4. Angew. Chem. Int. Ed. 2015, 54, 6447.

[45] N. Pienack, W. Bensch, In-situ monitoring of the formation of crystalline solids. Angew. Chem. Int. Ed. 2011, 50, 2014.

[46] E. Antonova, B. Seidlhofer, J. Wang, M. Hinz, W. Bensch, Controlling Nucleation and crystal growth of a distinct polyoxovanadate cluster: an in situ energy dispersive X-ray diffraction study under solvothermal conditions. Chem. Eur. J. 2012, 18, 15316.

[47] H. H.-M. Yeung, Y. Wu, S. Henke, A. K. Cheetham, D. O'Hare, R. I. Walton, In situ observation of successive crystallizations and metastable intermediates in the formation of metal-organic frameworks. Angew. Chem. Int. Ed. 2016, 55, 2012.

[48] K. M. O. Jensen, C. Tyrsted, M. Bremholm, B. B. Iversen, In situ studies of solvothermal synthesis of energy materials. ChemSusChem 2014, 7, 1594.

[49] S. Springer, A. Satalov, J. Lippke, M. Wiebcke, Nanocrystals and nanomaterials of isoreticular zeolitic imidazoate frameworsk. Microporous Mesoporous Mater. 2015, 216, 161.

[50] J.-P. Zhang, Y.-Y. Lin, X.-C. Huang, X.-M. Chen, Supramolecular isomerism within three-dimensional 3-connected nets: unusual synthesis and characterization of trimorphic copper(I) 3,5-dimethyl-1,2,4-triazolate. Dalton Trans. 2005, 3681.

[51] X.-C. Huang, J.-P. Zhang, X.-M. Chen, One-dimensional supramolecular isomerism of copper $(I)$ and silver(I) imidazolates based on the ligand orientation. Cryst. Growth Des. 2006, 5, 1194.

[52] J.-P. Zhang, X.-C. Huang, X.-M. Chen, Supramolecular isomerism in coordination polymers. Chem. Sov. Rev. 2009, 38, 2385. 
[53] A. F. Gualtieri, Synthesis of sodium zeolites from natural halloysite. Phys. Chem. Miner. 2001, 28, 719.

[54] J. Cravillon, S. Münzer, S.-J. Lohmeier, A. Feldhoff, K. Huber, $M$. Wiebcke, Rapid room-temperature synthesis and characterization of nanocrystals of a prototypical zeolitic imidazolate framework. Chem. Mater. 2009, 21, 1410.

[55] S. R. Venna, J. B. Jasinski, M. A. Carreon, Structural evolution of zeolitic imidazolate framework-8. J. Am. Chem. Soc. 2010, 132, 18030.
[56] T. Hikov, C. A. Schröder, J. Cravillon, M. Wiebcke, K. Huber, In situ static and dynamic light scattering and scanning electron microscopy study on the crystallization of the dense zinc imidazolate framework ZIF-zni. Phys. Chem. Chem. Phys. 2012, 14, 511.

[57] A. P. Hammersley, S. O. Svensson, M. Hanfland, A. N. Fitch, D. Häusermann, Two-dimensional detector software: from real detector to idealised image or two-theta scan. High Press. Res. 1996, 14, 235. 